Ecole d'Eté Systèmes Optiques

\title{
Les capteurs à fibres optiques
}

\author{
G. Boisde
}

Commissariat a l'Energie Atomique, DTA/LETI-DEIN/SAI, Centre d'Etudes Nucléaires de Saclay, F-91191 Gif-sur-Yvette cedex, France

\section{INTRODUCTION}

Les fibres optiques constituent un milieu de transmission d'excellente qualité. Les applications en télécommunications pour transmettre des signaux sur de grandes distances sont en continuel développement et commencent à être bien connues du grand public. Les technologies ainsi apparues depuis une dizaine d'années arrivent à maturité. Elles ont ainsi permis l'éclosion depuis 1980 de nouvelles applications des fibres dans le domaine des capteurs en utilisant les principes d'optique linéaire ou non linéaire.

Un capteur à fibre(s) optique(s) est ainsi défini comme un dispositif comprenant une ou plusieurs fibres optiques et qui détecte et transporte optiquement une information lumineuse représentative d'une (ou plusieurs) grandeur(s) à mesurer. Cette information peut être codée optiquement, être générée à partir d'ondes lumineuses circulant dans la fibre ; la lumière est, sauf pour la nouvelle génération de capteurs hybrides opto-alimentés, recueillie sans autre apport d'énergie extérieure que celui des phénomènes observés.

Les capteurs à fibres optiques (CFO) offrent un certain nombre d'avantages en regard des capteurs traditionnels : absence d'interférences électromagnétiques, sécurité intrinsèque (pas de risque d'étincelle), absence de problèmes liés à une terre, possibilité d'opérer (téléopération) et mesurer à distance (téléanalyse), souplesse géométrique (versatilité) et flexibilité, légèreté et robustesse, emploi des faibles niveaux de puissance optique, inertie chimique (selon le type de fibre), sensibilité accrue pour certaines applications, agencement aisé de dispositifs multifibres, adaptation en réseaux (multiplexage et mesure répartie), etc... Leurs inconvénients actuels sont surtout liés à la jeunesse de la technique (composants spécifiques onéreux, câbles et connecteurs optiques peu développés pour la télémesure, etc...) et à leur présence encore discrète sur le marché (une cinquantaine d'appareils CFO commercialisés dans le monde aujourd'hui).

En conséquence, ce n'est que récemment et postérieurement aux revues de Giallorenzi et coll aux USA /1/, de Arditty et coll en France /2/ (1982) que les capteurs à fibres optiques sont devenus un enjeu industriel. De nombreux articles de synthèse ont été publiés depuis 1985 tant pour les mesures physiques $/ 3-5 /$ que chimiques $/ 6-10 /$ tandis que deux ouvrages importants $/ 11-12 /$ viennent de paraître sur les sujets.

Cette revue succincte est destinée à fournir une synthèse de l'état de l'art des capteurs à fibres optiques en rappelant les principes de modulations de la lumière et les techniques utilisées les plus courantes (interférométrie, spectrométrie, réflectrométrie). Des exemples significatifs de mesures physiques seront donnés en précisant les principales applications et tendances actuelles. 


\section{1. - LES MODULATIONS DE LA LUMIERE}

Le capteur à fibres optiques (CFO) consiste ainsi en une (ou plusieurs) source(s) de lumière, un certain nombre de composants optiques (dont la fibre) pour conduire la lumière modulée, par la grandeur à mesurer, jusqu'à un (ou plusieurs) détecteur(s) éventuellement précédés de composants optiques appropriés. Quand il y a plusieurs sources de lumière, celles-ci peuvent être elles-mêmes modulées électroniquement dans le but de distinguer leurs influences respectives. On distingue les capteurs "extrinsèques", pour lesquels la modulation est externe à la fibre qui ne sert que de guide de lumière, et les capteurs "intrinsèques" pour lesquels la fibre est l'élément sensible en regard de la mesure à effectuer. Dans le domaine de la chimie on distingue les termes "actifs" et "passifs" avec l'introduction du terme "Optode" /13/ (ou Optrode) /14/ pour désigner l'élément sensible (généralement en extrémité de fibre optique). Ainsi les optodes passives (extrinsèques) sont des capteurs pour lesquels la fibre optique n'est qu'un guide de lumière qui "examine* le milieu à analyser. Avec les optodes actives l'interaction de la lumière avec le milieu environnant s'exerce dans la fibre elle-même ou à son extrémité. Dans ce cas elles sont généralement pourvues d'un réactif immobilisé sensible intégré au guide d'onde généralement par greffage chimique. Le schéma de principe d'une optode est ainsi donné en figure 1.

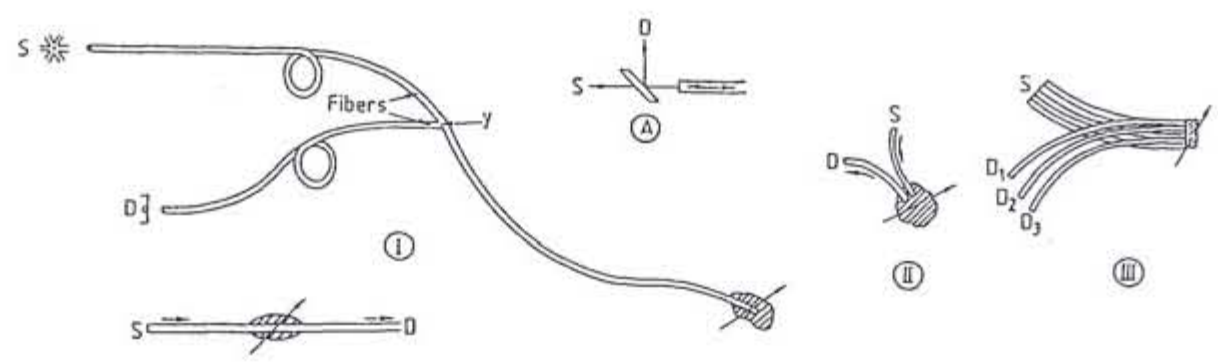

(11)

Figure 1: Schéma de principe d'une optode. Une source lumineuse S éclaire une fibre lou un faisceau de fibres) vers un (ou plusieurs) détecteur(s) D par l'intermédiaire d'une (ou plusieurs) fibre(s) réceptrice(s) de lumière. L'effet mesuré est généralement observé en extrémité de fibre(s) selon les montages I, II ou III. II peut être interne à la fibre ou occasionné par une variation des caractéristiques de la gaine optique $(M)$. Lorsque l'effet est analysé en retour sur une seule fibre, il est nécessaire d'utiliser un coupleur optique en forme $Y$ (schéma I) ou un ensemble optique à lame séparatrice (schéma A).

La solution typique sinusoïdale de l'équation de Maxwell pour une onde lumineuse se propageant dans la direction $\mathrm{z}$ pendant un temps t est :

$$
\begin{aligned}
& E x=E_{0} \exp [i(\omega t-k z)] \\
& H y=H_{0} \exp [i(\omega t-k z)]
\end{aligned}
$$

qui établit que le champ électrique Ex oscille sinusoïdalement dans le plan $x z$ et que le champ magnétique Hy oscille orthogonalement dans le plan yz. Une gamme de valeurs physiques peut être ainsi définie : 
- La fréquence $f$ et la fréquence angulaire ou la pulsation $\omega$ $\omega=2 \pi f=2 \pi / T$

- La longueur d'onde $\lambda$ et le nombre d'onde (son inverse) $1 / \lambda=f / v$

- La vitesse de la phase

$$
v=\omega / k
$$

- Le module $k$ du vecteur d'onde

$$
k=\omega / v=2 \pi / \lambda
$$

- L'indice de réfraction $n$ avec $c$ la vitesse de la lumière dans le vide

$$
n=c / v
$$

Ainsi l'intensité lumineuse par unité de surface (l) est proportionnelle au carré du champ électrique $1=\vee \varepsilon E^{2}$

où $\varepsilon$ est la permitivité électrique du milieu.

Par ailleurs, si deux ondes planes monochromatiques sont observées pour une valeur particulière $z_{0}$, elles prennent une forme oscillatoire

$$
\begin{aligned}
& E_{x}=e_{x} \cos \left(\omega t+\delta_{x}-k z_{\partial}\right) \\
& E_{y}=e_{y} \cos \left(\omega t+\delta_{y}-k z_{\partial}\right)
\end{aligned}
$$

où $E_{x}$ et $E_{y}$ sont dits polarisés linéairement respectivement dans les directions $O x$ et $O y$.

Si deux ondes lumineuses de même polarisation sont superposées en un point de l'espace, le résultat est une autre sinusoîde :

$$
\begin{aligned}
& e_{1}=E_{1} \cos \left(\omega t+\varphi_{1}\right) \\
& e_{2}=E_{2} \cos \left(\omega t+\varphi_{2}\right)
\end{aligned}
$$

et le champ résultant $E_{1}$ à ce point pour $E_{1}=E_{2}=E$ est :

$$
\left(E_{T}\right)^{2}=4 E^{2} \cos ^{2}\left(\varphi_{2} \cdot \varphi_{1}\right) / 2
$$

Les variations d'intensité à une distance donnée montrent des franges d'interférence. Ce phénomène est la base de l'interférométrie.

On peut ainsi distinguer quatre types de modulation de la lumière :

$1 \%$ La modulation d'amplitude caractéristique de la variation d'intensité de la lumière (nombre de photons). Elle est évaluée en puissance optique $(P)$ et souvent donnée en intensité relative (spectrométrie de fluorescence par exemple) ou en unités d'atténuation $A$, en absorbance $a$, ou son inverse (1/a) la transmittance.

$$
\mathrm{A}(\text { en décibels })=10 \mathrm{Log}(\mathrm{Po} / \mathrm{P})=10 \mathrm{a}
$$


Ce type de mesure requiert généralement une référence (de préférence interne au dispositif) ou un système de balance différentiel afin de compenser les fluctuations de la source, les pertes optiques, etc...

$2 \%$ La modulation de phase $(\varphi)$ est l'équivalent d'une mesure de temps de vol des photons. Elle est caractéristique d'une variation de trajet optique pour une fibre monomode, ou d'allongements relatifs des trajets optiques de plusieurs modes (figure de speckle) pour une fibre multimode lorsqu'on utilise comme source lumineuse une lumière monochromatique (laser). Généralement on mesure en effet relatif (franges d'intertérences) entre une voie de référence et une voie de mesure de lumière cohérente monochromatique. La diffraction de la lumière peut être considérée comme un cas limite d'intertérences multiples lorsque l'espacement entre les sources devient infiniment petit.

$3 \%$ La modulation en longueur d'onde $(\lambda)$ qui correspond à des variations de position spectrale (énergie) ou de largeur spectrale, donc à une statistique temporelle. Un appareil utilisant la modulation en longueur d'onde comporte ainsi une source à large spectre, un modulateur de couleur, des éléments de spectrométre (prismes, réseaux, filtres), un détecteur et un système de traitement de signal. La résolution dépend des caractéristiques du détecteur, de la stabilité du modulateur et du spectrométre, etc...

Cette modulation est le plus souvent associée à la modulation d'intensité (spectrométrie, pyrométrie, etc...).

$4 \%$ La modulation de polarisation caractéristique des changements de birefringence et donc de variations d'ellipticité (spin des photons). Dans les montages polarimétriques on mesure la différence de phase déduite de l'état de polarisation de deux modes polarisés d'une même fibre monomode. Dans certains montages on mesure la rotation de la polarisation en fonction de la grandeur d'influence. Les propriétés optiques de polarisation d'une fibre peuvent être influencées par l'action de champ électrique ou magnétique. Deux effets actifs principaux illustrent ce phénomène (figure 2) :

- I'effet électro-optique : lorsqu'un champ électrique est appliqué à un milieu optique le matériau devient linéairement biréfringent avec un mouvement limité des électrons dans la direction du champ en comparaison de la direction orthogonale. Le retard de phase introduit est proportionnel au champ (effet Pockels) ou au carré du champ (effet Kerr).

- l'effet magnéto-optique : l'effet de polarisation d'un champ magnétique longitudinal H sur un matériau optique provoque la rotation d'un angle $\theta$ de la direction de polarisation d'une lumière polarisée linéairement à l'entrée de ce matériau (effet Faraday).

$$
\theta=\underset{0}{\mathrm{~V}} \int_{\mathrm{H} . \mathrm{dl}}^{\mathrm{L}}
$$

où $V$ est la constante de Verdet.

$5 \%$ On admet parfois un cinquième type de modulation : la modulation temporelle significative de l'évolution d'un signal au cours du temps (chronométrie). Un exemple typique est donné en fluorimétrie temporelle en s'appuyant sur la durée de vie du fluorophore. Elle est à distinguer de la modulation temporelle utilisée lors du traitement de signal pour la plupart des capteurs à fibres optiques (fréquence de quelques $\mathrm{Hz}$ à quelques $\mathrm{MHz}$ ). 


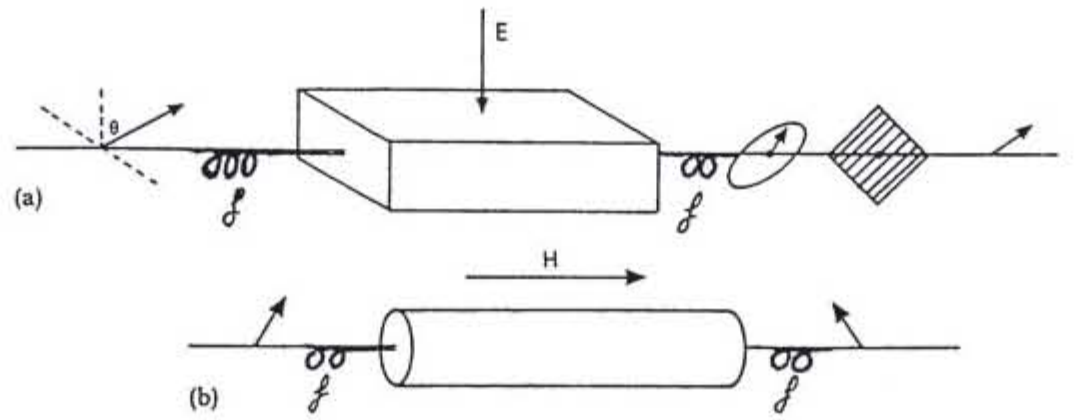

Figure 2 : Effets de polarisation a) électro-optique b) magnéto-optique ; $f=$ fibre optique.

Ces modulations sont le plus souvent combinées et fournissent toute une gamme de techniques nouvelles qui, associées aux fibres optiques, apportent des possibilités originales et des avantages essentiels pour la mesure.

\section{2. - LES TECHNIQUES}

\section{1. - L'intertérométrie}

La variation de phase de la lumière peut être observée selon les techniques interférométriques habituelles. Quatre architectures sont bien connues (figure 3) : Mach Zehnder, Michelson, Sagnac et Fabry Perot. Elles nécessitent l'emploi de fibres monomodes.
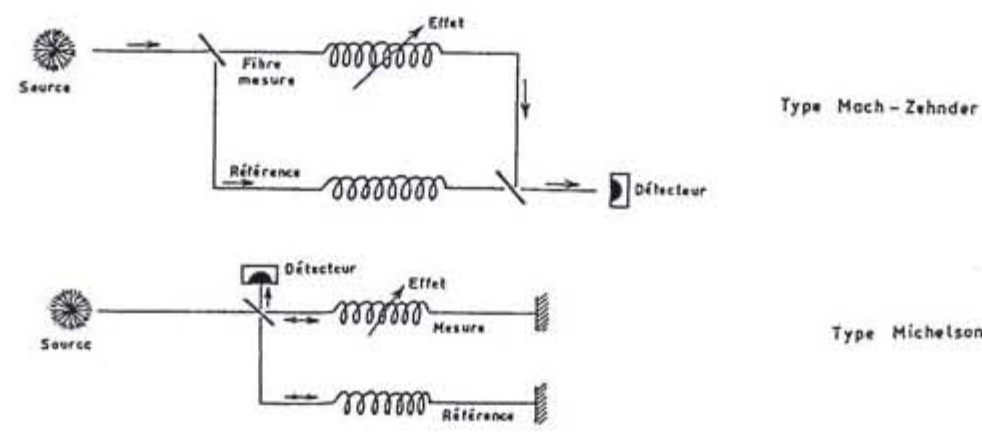

Type Michetson

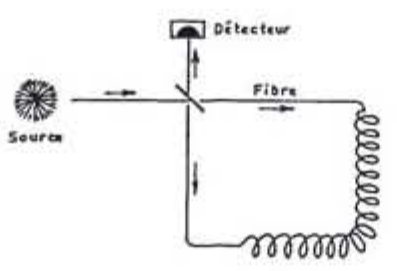

Type Sagnoc

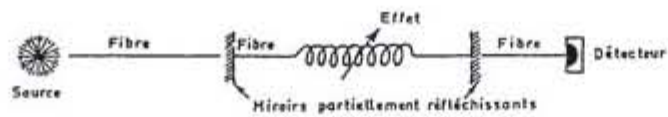

Type. Fabey-Perot

Figure 3 : Techniques interférométriques à fibres optiques. 
Dans l'interferométrie à deux ondes (Mach Zehnder et Michelson) les deux faisceaux guidés sont recombinés et mélangés de manière cohérente pour produire des interférences observables sur deux sorties $\left(I_{1}\right.$ et $\left.I_{2}\right)$. On peut montrer $/ 15 /$ que le contraste (ou visibilité) d'interférence est donné par :

avec

$$
V=(I \max -I \min ) /(I \max +I \min )
$$

et

$$
I_{1}=I_{0}\left[1-V \cos \left(\varphi_{1}-\varphi_{2}\right)\right]
$$

$$
I_{2}=I_{0}\left[1+V \cos \left(\varphi_{1}-\varphi_{2}\right)\right]
$$

Le maximum d'intensité est obtenu lorsque les bras de l'interférométrie sont en quadrature, d'où l'usage de modulateur dans l'un des bras (généralement la référence). Une technique alternative utilise deux sources de longueurs d'onde légèrement différentes afin d'accroître la dynamique de mesure /16/. L'intertérométrie réciproque (Sagnac) est du type bi-onde, les deux faisceaux circulant dans des directions opposées. II est utilisable pour des effets non symétriques et à la base de nombreux capteurs gyroscopiques à fibres optiques /17/.

Le plus courant des intertérométres à multiples faisceaux est le Fabry Perot. II utilise également de la fibre monomode. Lorsqu'on éclaire une fibre multimode avec de la lumière cohérente on observe en sortie de fibre une granularité due aux interférences intermodales d'où la figure de speckle en champ lointain. Des effets de contraintes sinusoïdales imposées à la fibre induisent une modification des constantes de propagation qui peuvent être analysées en fonction des variations de paramètres extérieurs (vibrations, température, etc...) $/ 5,18$ /.

L'interférométrie en lumière blanche est une nouvelle technique alternative pour laquelle la cohérence de la source (diode électroluminescente) est moindre que celle d'un laser. L'interférence est observée seulement lorsque les différences de parcours optique dans l'interférométre sont bien balancées. Cette technique est utilisable avec des fibres multimodes $/ 19 /$ ou à 2 modes $/ 20 /$.

On peut noter que les variations de polarisation linéaire, circulaire, sont généralement étudiées avec des montages de type interférométrique en mesurant à la fois la phase et l'état de polarisation d'une fibre birefringente sensible à la grandeur à mesurer.

\section{2. - La spectrométrie}

C'est un ensemble de techniques qui exploitent depuis l'ultraviolet jusqu'à l'infrarouge (à ce jour de $0,25 \mu \mathrm{m}$ à $5 \mu \mathrm{m}$ avec des fibres optiques) les variations spectrales d'un transducteur.

\subsection{1. - Absorption}

La mesure d'absorption est basée sur la loi de Beer-Lambert pour laquelle l'absorbance $\left(a_{\lambda}\right)$ d'un faisceau lumineux d'intensité intiniale lo réduite à I après passage sur une distance $\ell(\mathrm{en} \mathrm{cm})$ est proportionnelle à la concentration $\mathrm{C}$ de l'espace à mesurer :

$$
\text { (a) }=\log (10 / I)=\varepsilon_{\lambda} \text { lc. }
$$

où $\varepsilon_{\lambda}$, le coefficient d'absorption molaire, est une constante de proportionnalité (dépendante de la longueur d'onde $\lambda$ ). Trois types de mesure sont généralement adoptés : la mesure à une longueur d'onde (le plus souvent au pic d'absorption), la mesure à deux longueurs d'onde, l'une servant de référence interne, la mesure multilongueurs d'onde en particulier avec un réseau et une barrette de 
photodiodes comme détecteurs. Ce dernier dispositif permet la mesure en temps réel déportée par fibres optiques (téléanalyse) /21/.

\subsection{2. - Réflectance}

Lorsque la lumière rencontre une discontinuité d'indice de réfraction, il y a réflexion. La réflexion spéculaire sur des surfaces réfléchissantes (faible absorption) est utilisée pour un nombre de capteurs, de mesure physique (déplacement, pression, etc..). La réflexion diffuse sur des espèces absorbantes de concentration (C) est généralement étudiée suivant la fonction de Kubelka-Munk :

$$
F(R)=\varepsilon_{\lambda} \cdot C / S=(1-R)^{2} / 2 R
$$

où $S$ est un coefficient de diffusion et $\mathrm{R}$ la réflectance.

\subsection{3. - Luminescence}

Une substance éclairée par une radiation réémet de la lumière, soit par une cascade de désactivation non radiative avec conversion en énergie thermique, soit par émission d'un second photon, soit par transfert interespèces avec un "accepteur" qui ensuite émet un second photon. Les photons réémis sont moins énergétiques et leurs longueurs d'onde sont décalées vers le rouge par rapport à la longueur d'onde d'excitation. Cette propriété permet d'obtenir de grandes sensibilités par cette technique. Les durées de vie de ces photons, en fluorescence de l'ordre de $1 \mathrm{~ns}$ à $10 \mathrm{~ms}$, sont largement accrues en phosphorescence (>100 ms). On mesure généralement :

- soit l'intensité de la fluorescence du fluorophore (élément sensible)

$$
F=k \cdot \theta_{F} \cdot P o . \varepsilon \mathcal{P C}
$$

où Po est la puissance de la lumière incidente, $\theta_{\mathrm{F}}$ le rendement quantique de fluorescence, $\mathrm{k}$ un coefficient géométrique de proportionnalité,

- soit l'exaltation ou la diminution de la fluorescence (quenching) en présence d'espèces ioniques étrangères au fluorophore

$$
\mathrm{Fo} / \mathrm{F}=1+\mathrm{Ksv} \cdot \mathrm{Cq}
$$

ou Cq est la concentration du "Quencher" et Ksv la constante de Stern-Volmer,

- soit la mesure de transfert d'énergie entre un "donneur" et un "receveur" fluorescent, le paramètre de mesure étant la diminution de l'émission du donneur ou la régénération de la fluorescence du receveur.

Dans certains cas, on assure la sélectivité de la méthode par des mesures temporelles liées à la durée de vie d'un complexe fluorophore - espèce à analyser. Ce type de mesure est à l'origine de la spectrométrie laser à résolution temporelle expérimentée avec fibres optiques comme représenté en figure $4 / 22 /$. 


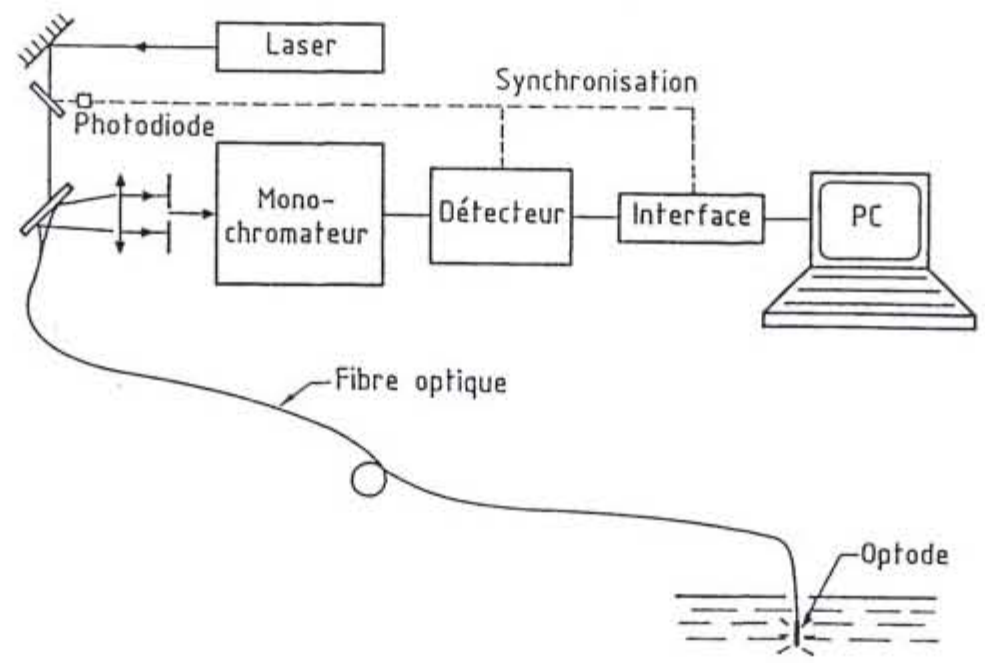

Figure 4 : Principe de la spectrofluorimétrie laser à fibres optiques et résolution temporelle.

\subsection{4. - Diffusion et spectrométrie Raman}

La diffusion de la lumière par les particules du milieu peut être élastique, comme la diffusion Ragleigh (sur des particules de l'ordre de $\lambda / 10$ ) ou la diffusion Mie (particules supérieures à $\lambda$ ), ou non élastique (diffusion Raman) lorsqu'un processus d'absorption accompagne la diffusion et cause un changement d'énergie vibrationnelle et/ou rotationnelle (de polarisation) des molécules. L'intensité de l'émission Raman à plus courte longueur d'onde (anti-Stokes) et surtout plus grande longueur d'onde (raies Stokes) est proportionnelle à la concentration des espèces à mesurer et spécifique des liaisons chimiques. De nombreux travaux dans ce domaine ont été réalisés en France pour la mesure "in situ*. Ils ont conduit à des conceptions d'optodes originales /23/. Cette technique avec transformée de Fourier est l'objet de nouvelles recherches en proche IR $(1,064 \mu \mathrm{m})$.

\section{3. - Réfractométrie et spectroscopie d'onde évanescente}

Une lumière frappant l'interface de deux milieux transparents d'indices de réfraction différents peut être partiellement réfléchie et partiellement transmise. Cette propriété peut être mise à profit pour réaliser des capteurs à fibres optiques à gaine dénudée; la variation d'intensité de la lumière dans le guide d'onde est ainsi influencée par la variation d'indice de réfraction du milieu environnant (réfractométrie). Si la lumière frappe l'interface en venant du milieu à plus faible indice, l'effet est appelé réflexion externe. Dans le cas contraire l'effet est la réflexion interne.

La spectroscopie d'onde évanescente utilise le fait que la lumière peut rester dans un guide d'onde (fibre) lorsque l'angle d'incidence $(\theta)$ du rayon lumineux - à l'interface guide d'onde - gaine optique (liquide à mesurer) est légèrement supérieur à l'angle critique $\left(\theta_{\mathrm{C}}\right)$. La lumière pénètre légèrement à l'interface selon une fraction de longueur d'onde dans la gaine, laquelle peut être le siège de réactions chimiques. Cette pénétration schématisée en figure 5 , à distance $z$ du guide, dépend des conditions de liaison à l'interface. Le champ électrique $(E)$ de l'onde évanescente est :

$$
E=E_{0} \exp (-z / d p)
$$


où $\mathrm{dp}$ est la profondeur de pénétration déterminée par la diminution de l'amplitude du champ électrique à $1 / \mathrm{e}$ de sa valeur à la surface

$$
d p=(\lambda / 2 \pi) \cdot\left(n_{1}^{2} \sin ^{2} \theta \cdot n_{2}^{2}\right)^{-1 / 2}
$$

pour $\mathrm{n}_{1}>\mathrm{n}_{2}$ avec $\theta$ l'angle du mode guidé à l'interface guide d'onde liquide à mesurer. Des applications nombreuses ont été trouvées, en particulier pour réaliser des biocapteurs /24/ en couplant le phénomène d'ondes évanescentes avec la spectroscopie (fluoroimmuno essais etc...).

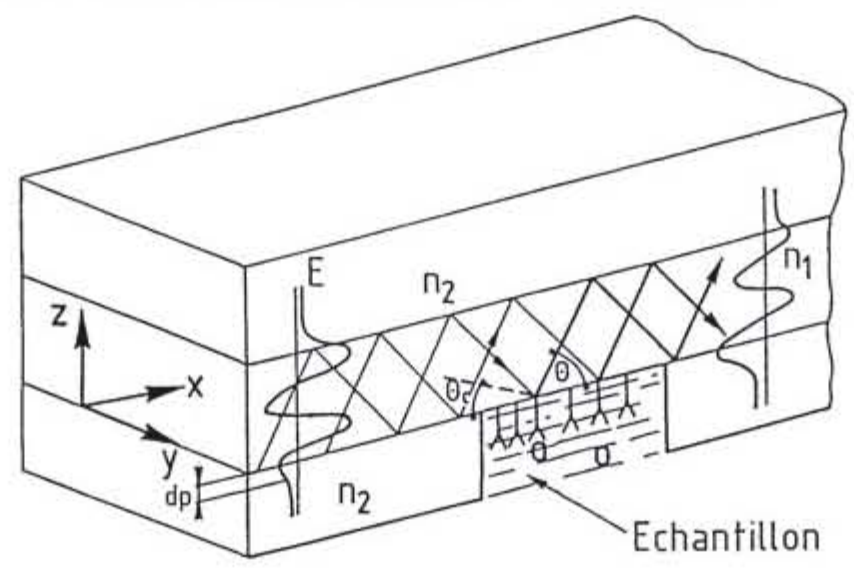

Figure 5 : Configuration schématique d'un guide d'onde planaire symétrique.

L'intensité de l'onde évanescente peut être accrue par un phénomène d'oscillation collective d'un plasma d'électrons libres à l'interface d'une couche diélectrique-métal /25/. Cet effet appelé résonance de plasma de surface (surface plasmon résonance) se produit lorsque la lumière est totalement réfléchie dans le diélectrique. II engendre une forte variation du coefficient de réflexion pour un angle critique (angle de résonance). La modification des propriétés de l'échantillon décalant cet angle permet des mesures d'interaction échantillon-guide d'onde de grande sensibilité.

Les techniques de réalisation de guides d'onde planaires et d'optique intégrée sont des voies très prometteuses pour ces types de capteurs.

\section{4. - Réflectrométrie}

La réflectrométrie utilise les principes de la rétrodiffusion de la lumière en extrémité de fibre. Une impulsion lumineuse transmise dans la fibre est reçue en retour come un écho depuis son extrémité. Le temps de propagation du signal dépend linairement de la longueur de la fibre (vitesse de la lumière dans le guide d'onde). L'affaiblissement linéïque (atténuation) est établi relativement à cette longueur. Une variation d'atténuation le long de la fibre peut être repérée en amplitude et en temps de transit (donc en distance de parcours dans la fibre). La figure 6 illustre ce type de mesure. Cette technique et ses dérivées sont connues sous le nom OTDR (Optical time domain reflectometry), POTDR (Polarisation OTDR) FOTDR (fréquency OTDR) et peuvent être couplées avec la mesure des variations d'indices de réfraction et la spectrométrie. Elles permettent d'accéder à une structure en réseau par un multiplexage temporel (réflectrométrie résolue dans le temps) /15/. 


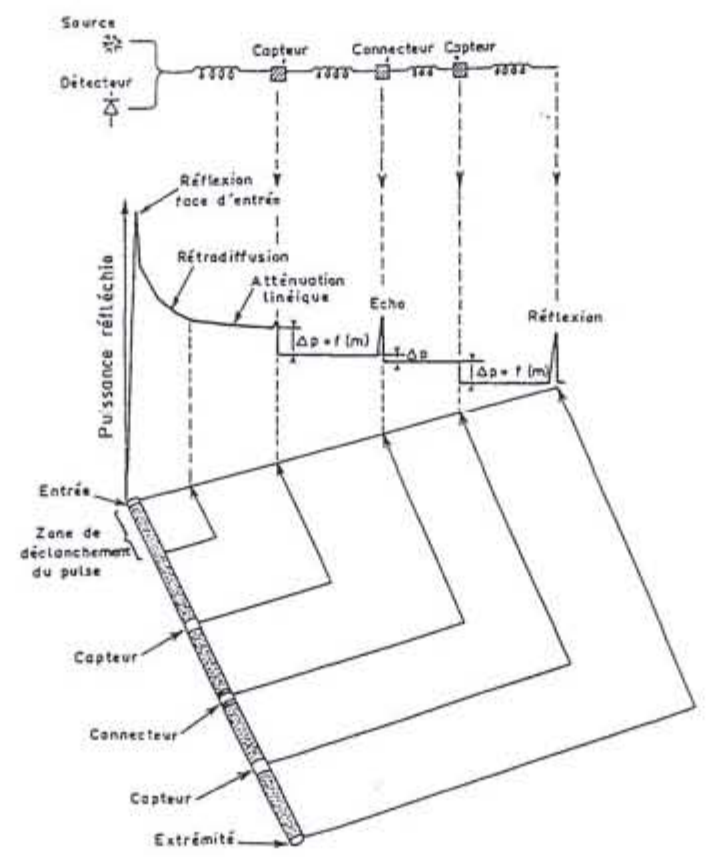

Figure 6 : Principe de la mesure "répartie" à fibres optiques par réflectométrie optique.

\section{3. - QUELQUES EXEMPLES DE CAPTEURS A FIBRES OPTIQUES}

Aujourd'hui on constate une augmentation considérable du nombre de publications dans le domaine des capteurs à fibres optiques avec une augmentation de 25 à $40 \%$ par an suivant les applications depuis 1983 (date de la première conférence internationale OFS sur le sujet). En conséquence les exemples cités ici, quoique significatifs, ne permettent d'avoir qu'une vue incomplète /1 - 12/. Une étude récente portant sur 2700 brevets de capteurs optiques donne une indication globale de leur répartition par ordre d'importance /26/. Elle est représentée en figure 7.

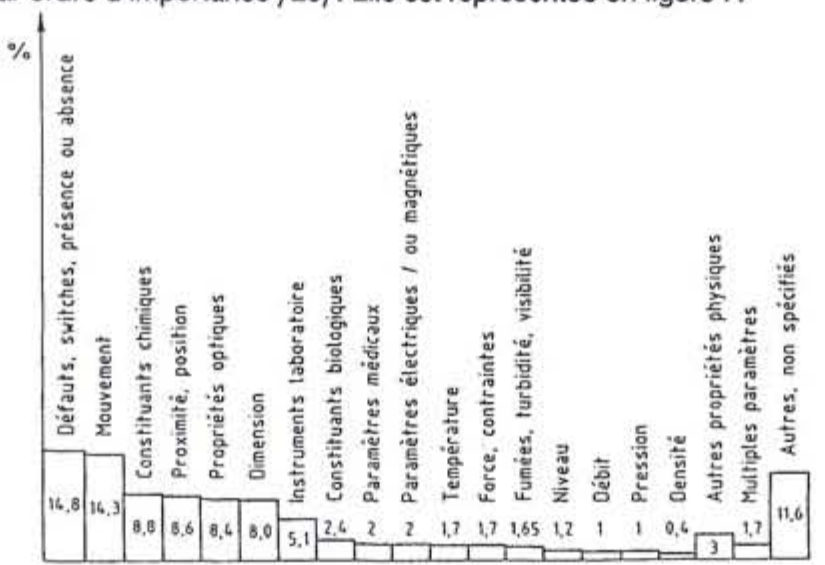

Figure 7 : Répartition de 2700 brevets "Capteurs optiques" par type de mesure. (Référence 26). 


\section{1. - Capteurs de mesures physiques}

\subsection{1. - Forces mécaniques}

II s'agit de mesures linéiques, angulaires, cinématiques, massiques et manométriques.

Parmi les mesures linéiques (proximité, distance, position, déplacement, niveau) les systèmes "tout ou rien" ont fait jusqu'ici l'objet des plus nombreuses applications, soit par mesure directe (couplage de fibres par transmission) soit en mesure reflex (cible coopérante) ou rétrodiffusée. Le codage optique absolu ou incrémental est basé sur une modulation d'amplitude (intensité) à l'aide d'une règle ou d'un disque à fentes prépositionnées. La réflexion optique permet des mesures de déplacement en fonction de la distance de la cible. Ainsi, selon la figure 8 , deux fibres axialement séparées pour fournir deux caractéristiques de collimation, permettent de réaliser un capteur sans contact à référence intrinsèque (précision $10 \mu \mathrm{m}$ sur une gamme de 3 millimètres) /27/. L'usage d'un référençage à deux longueurs d'onde a également été développé $/ 28$. L'interférométrie en optique intégrée apporte également des solutions avantageuses pour des mesures de l'ordre du nanomètre. En mesure de niveau, on exploite la variation d'indice de réfraction, la réflexion totale sur un prisme, la mesure photométrique et les méthodes dérivées de mesures de déplacement par télémétrie laser. Dans ce dernier cas les schémas de montage sont généralement de type interférométrique et les méthodes incluent la mesure du temps de parcours d'une impulsion, la mesure de déphasage entre une onde de référence et l'onde réfléchie, la modulation de fréquence.
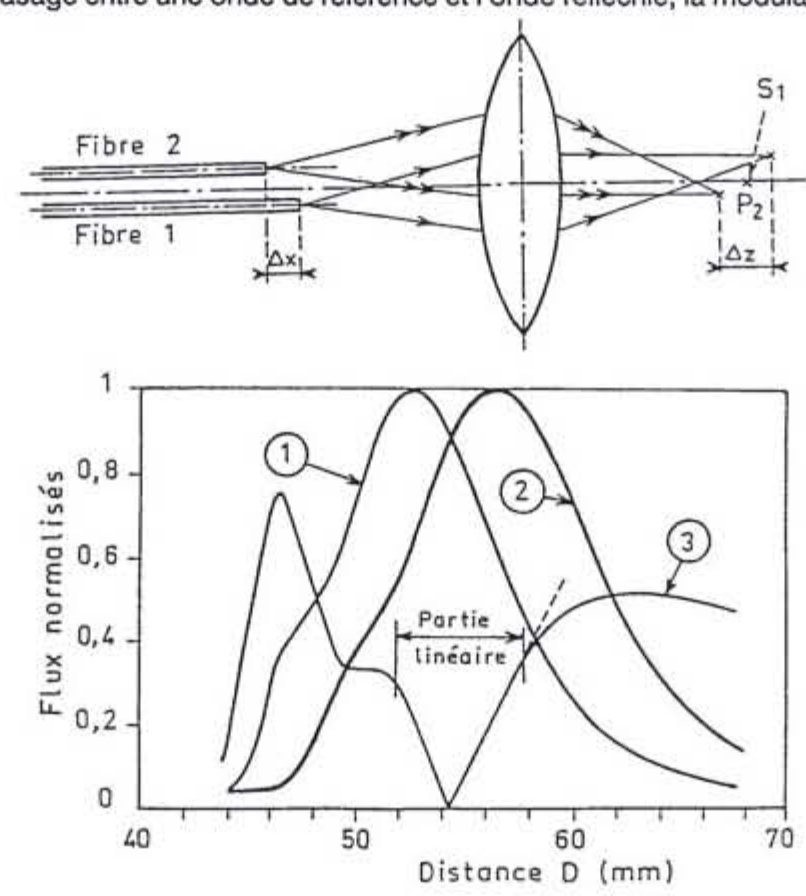
(1) $\varphi_{1}$
(2) $\varphi_{2}$
(3) $\frac{\left|\varphi_{1}-\varphi_{2}\right|}{\left(\varphi_{1}+\varphi_{2}\right)}$

Figure 8 : Principe d'un capteur de déplacement sans contact, à référence intrinsèque. 
Deux grandes familles de capteurs angulaires ont été développées, l'une concerne les codeurs optiques, l'autre les intertérométres particulièrement de type Sagnac (gyroscope à fibres).

Dans le cadre des mesures cinématiques (vitesse, accélération, vibrations) on peut citer pour des accéléromètres, la variation d'amplitude par couplage de deux fibres face à face, par mesure de vibration d'une lamelle mobile en bout de fibre (figure 9) ou la modulation de polarisation par utilisation de l'effet photoélastique (birefringence induite sous contrainte). En mesure de vitesse, l'effet Doppler (écart de fréquence entre la lumière incidente et la lumière diffusée) en détection directe ou hétérodyne est le plus souvent utilisé.

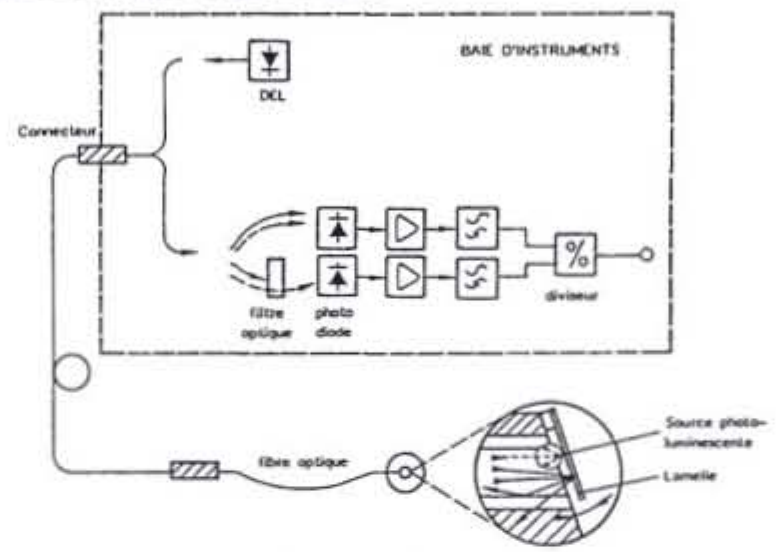

Figure 9 : Schéma de principe d'un accéléromètre à fibre optique multimode.

Dans la gamme des capteurs de débit on citera surtout la réflexion optique sur un corps mort dans un effet Vortex et les capteurs double phase (eau, air) par variation d'indice de réfraction en extrémité de fibre $/ 29 /$.

Les capteurs manométriques (pression statique, acoustique, pression différentielle, contraintes) ont fait l'objet de nombreux développements. Dans les capteurs extrinsèques, on mesure la déformation d'une membrane sous l'influence de la pression par une variation d'amplitude du signal réfléchi. Des variations de pression sont aussi mesurables par effet photoélastique (capteurs à modulation de polararisation). Une variation d'amplitude est aussi provoquée par les déformations de la fibre ellemême (création de microcourbes) sous l'influence d'une pression (figure 10). Dans ce capteur "intrinsèque", la périodicité de la perturbation est choisie pour un couplage maximum entre les modes radiés. L'intertérométrie est surtout utilisée pour les mesures de pression acoustique (hydrophones) et de pression différentielle.
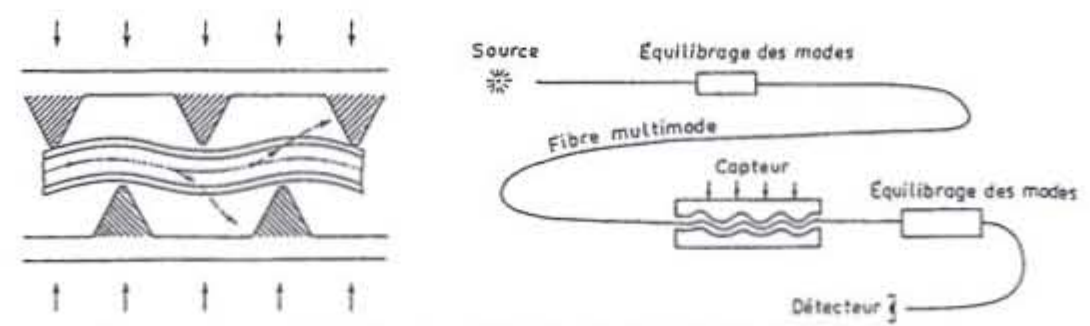

Figure 10: Principe d'un capteur de pression par création de microcourbures. 


\subsection{2. - Forces électriques et magnétiques}

Les capteurs interférométriques à fibres optiques monomodes basés sur la variation d'indice du mode lors de la compression de la fibre enrobée d'un polymère piézoélectrique permettent des mesures de champ électrique à partir du $\mathrm{kV} /$ mètre de fibre $/ 30$. L'effet Pockels, variation linéaire de l'indice optique avec le champ électrique, a été utilisé dans des modulateurs électro-optiques à niobiate de lithium. Pour une mesure de champ dans une direction connue, le cristal de germanate de bismuth a été proposé $/ 31$. Enfin, avec un détecteur polarimétrique, l'effet Faraday permet de réaliser un capteur de courant électrique dans une gamme de 0.1 à $1000 \mathrm{~A}$. L'optique intégrée apporte aussi à ce type de mesure des solutions originales. La transduction magnétostrictive (mesure de champ magnétique par effet Faraday) est généralement associée à des mesures de contraintes de la fibre optique. Avec un montage intertérométrique la sensibilité obtenue peut atteindre $10^{-11}$ tesla pour un mètre de fibre.

\subsection{3. - Température}

De très nombreux capteurs de température ont été décrits pour une large gamme d'utilisation (- 160 $a+2000^{\circ} \mathrm{C}$ ). Certains pyromètres reposent sur les caractéristiques d'émissivité du rayonnement infrarouge (loi de Planck) avec des mesures à une ou plusieurs longueurs d'onde. La fibre de verre fluoré (utilisable jusqu'à $5 \mu \mathrm{m}$ ) permet des résolutions d'environ $3^{\circ} \mathrm{C}$ aux alentours de zéro $/ 32 /$. D'autres thermomètres extrinsèques sont basés sur le rayonnement d'un corps noir en bout de fibre (principe NBS figure 11), sur les variations de propriétés optiques des cristaux liquides (gamme 0 $200^{\circ} \mathrm{C}$ ), sur les variations de caractéristiques d'absorption (en intensité ou déplacement de bande spectrale) de verres dopés au néodyne ou à l'europium, sur des variations de luminescence par analyse spectrale de la fluorescence de terres rares ou d'un cristal AsGa ou par analyse temporelle d'un phénomène de phosphorescence. Une autre catégorie repose sur les modifications de birefringence d'un cristal (niobate de lithium) ou sur le déphasage entre deux modes de propagation d'une fibre biréfringente. Enfin, certains capteurs sont de type interférométrique Fabry Perot ou Michelson. La réflectrométrie optique (OTDR et dérivés) permet d'aborder la classe des thermomètres répartis, distribués et des réseaux de capteurs $/ 5 /$. Une récente bibliographie bien documentée fait récemment le point sur le chapitre des capteurs de température à fibres optiques $/ 33 /$.

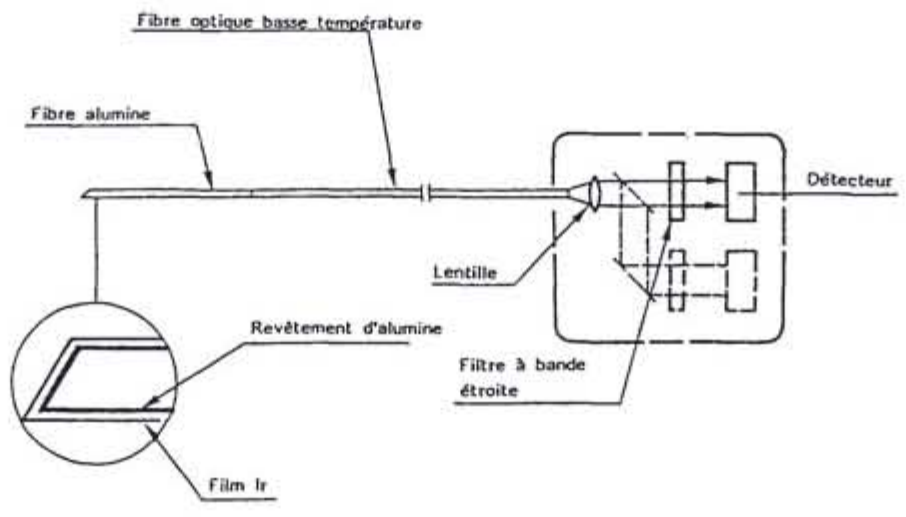

Figure 11 : Principe du capteur NBS (National Bureau of Standards) pour haute température $\left(2000^{\circ} \mathrm{C}\right)$. 


\subsection{4. - Forces Nucléaires}

Les fibres optiques plastiques sont couramment utilisées comme détecteurs de particules /34/. La détection du rayonnement ionisant est faite avec une substance scintillante sensible incorporée à la fibre ou en extrémité de la fibre. L'introduction dans une fibre de dopants fluorescents ou à variation de spectre sous l'influence des radiations nucléaires est également utilisé $/ 35 /$. Des phénomènes de thermoluminescence (régénération thermique de fibres irradiées), de variations relatives d'atténuation à deux longueurs d'onde ont aussi fait l'objet d'investigations variées. En particulier des verres dopés au cérium, au plomb ou au phospore peuvent faire de bons capteurs intégrés. Une fibre à liquide organique avec substance radiocrö̈que a également été utilisée pour des doses de 0,1 à $10^{5}$ rads $/ 36 /$. Récemment des dosimètres à fibres optiques ont été conçus en technique OTDR $/ 37 /$. Leur sensibilité est d'environ 10 rads $(0,1$ Gray)pour une résolution localisée de 2 centimètres. Ces dosimètres sont réalisés pour des applications thérapeutiques.

\section{2. - Capteurs de mesures chimiques}

\subsection{1. - Optodes passives (extrinsèques)}

L'usage de ces optodes est généralement réservé aux techniques de spectrophotométrie, de diffusion de lumière, de spectrofluorimètrie, de chimie et bio-luminescence et de spectrométrie Raman. En absorptiométrie on utilise soit des sondes de mesure in situ, soit des cellules à circulation. La fibre optique, de faible dimension transverse, permet des miniaturisations avantageuses et donc des rapports volume mesuré $(v)$ parcours optique $(l)$ très performants $(v / l=3$ $\mathrm{ml} . \mathrm{m}^{-1}$ ). Dans l'infrarouge des adaptations de cellules à grand parcours ont permis la mesure de $\mathrm{CH}_{4}$ ou le contrôle en ligne de traces d'uranium /8/. Egalement dans l'infrarouge des optodes multifibres en verres fluorés ou silice ont permis la mesure des sucres à travers la peau des fruits.

Une des applications les plus remarquables des fibres optiques est la spectrofluorimétrie déportée à résolution temporelle (figure 4), une méthode sélective de plus en plus utilisée pour des mesures d'environnement. Les architectures d'optodes pour spectrofluorimétie et spectrométrie Raman déportées ont commencé à faire l'objet de nombreux travaux de modélisation, aussi bien pour l'agencement des systèmes multifibres /38/ que pour la détermination de l'effet des espèces absorbantes en fluorescence.

En milieux industriels, les applications des optodes passives ont surtout porté sur les gaz $\left(\mathrm{CH}_{4}\right.$, $\mathrm{C}_{3} \mathrm{H}_{8}$ ), les espèces chimiques nucléaires (uranium, plutonium) $/ 39 /$, le contrôle de produits pétroliers, des fermentations alcooliques, des produits alimentaires, la surveillance de l'environnement, le contrôle de l'action chlorophyllienne, etc.. En milieu médical, ces techniques sont adaptées pour l'oxymétrie (mesure de l'hémoglobine), l'inspection des artères et du coeur (mesure du NADH par fluorescence) via un catheter. La spectrométrie à transformée de Fourier (IR, Raman) et à fibres optiques est aussi une technique en pleine évolution.

\subsection{2. - Optodes actives}

Les optodes actives peuvent être de type extrinsèque ou intrinsèque, ces dernières utilisant le plus souvent les principes de mesure des ondes évanescentes ou de l'interférométrie. Elles obéissent à la loi des équilibres chimiques enre un réactif, par exemple un indicateur $\mathrm{HI}$ et les produits de réaction, par exemple l'ion $\mathrm{H}^{+}$et la base conjuguée $\mathrm{I}^{-}$

$$
\mathrm{HI} \rightleftharpoons \mathrm{H}^{+}+\mathrm{I}^{-}
$$


A partir de la loi d'action de masse on définit la constante d'équilibre $\mathrm{K}$ :

$$
K=\left[\mathrm{H}^{+}\right] \cdot\left[\mathrm{I}^{-}\right] /[\mathrm{HI}]
$$

Depuis 1977, date des travaux du NIH (USA) /40/ de nombreuses optodes de mesure de pH ont été réalisées dans le monde. La mesure à deux longueurs d'onde (avec rétérence à 830 nanomètres) est aujourd'hui classique. Elle est en compétition avec la fluorescence (emploi d'un fluorophore). Les travaux les plus récents portent sur limmobilisation des réactifs soit placés en extrémité de fibre, soit par greffage de polymères sur la fibre elle-même. Un exemple de polymère greffé sur silice est donné en figure $12 / 41 /$. Une tendance actuelle consiste à réaliser plusieurs capteurs miniaturisés dans un même catheter comme illustré en figure $13 / 42 /$ pour la mesure simultanée de $\mathrm{pH}, \mathrm{PCO}_{2}$ et $\mathrm{pO}_{2}(\mathrm{CDI}$ - système 400). Une autre voie consiste à immobiliser plusieurs indicateurs sur le même support, par exemple pour une mesure $\mathrm{pH}$ large gamme $/ 43,44 /$.

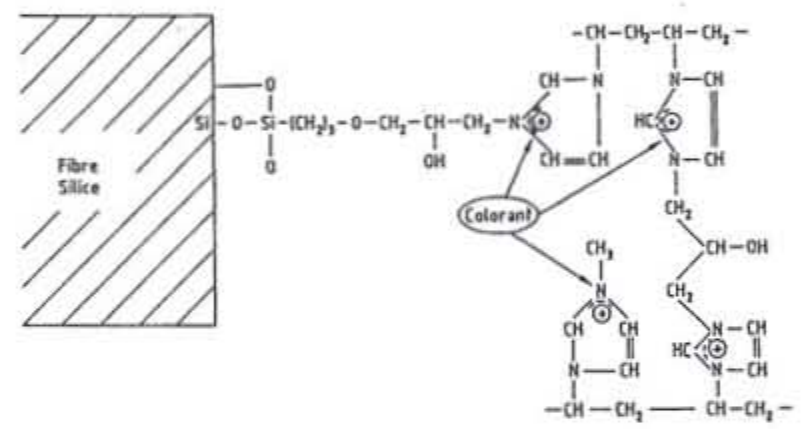

Figure 12 : Exemple d'immobilisation d'un polymère (poly-vinylimidazole) greffé sur fibre optique de silice pour mesure de $\rho H$. La liaison avec le colorant est de type lonique.

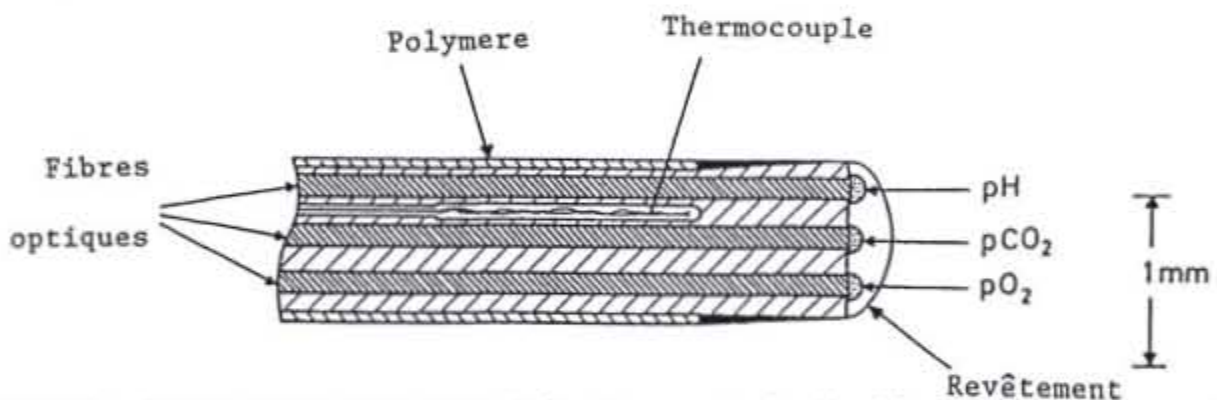

Figure 13 : Capteur chimique de type cathéter pour mesure de $\mathrm{pH}, \mathrm{pCO}_{2}$ et $\mathrm{pO}_{2}$ dans une artere (référence 42).

De nombreuses optodes incluent des membranes qui agissent soit comme élément de sélectivité des ions à doser, soit comme support polymérique. Les procédures d'immobilisation sont basées sur :

- l'adsorption : entre l'indicateur et le polymère support. II s'agit par exemple d'une liaison hydrophobe par interaction de type donneur-accepteur. Les polymères supports les plus courants sont la cellulose, les membranes PVC, silicone ou de polyéthylène glycol, les résines échangeuses d'ions telles que XAD2 ou XAD4 /41, 45/. 
- la liaison ionique qui met en jeu des interactions électrostatiques entre un indicateur chargé et un support chargé de type cationique ou anionique. II a la particularité de posséder des parties à la fois hydrophobes et ioniques. L'exemple de la polyvinylimidazole greffée sur fibre et associée à des phénolphtaleïnes comme indicateurs (figure 12) est significatif de cette technique.

- la liaison covalente qui nécessite une chimie plus complexe à partir soit de gels (polyacrylamide, alcool polyvinylique, etc..) soit de polymères (chaines amides ou imides) greffés sur fibres $/ 46 /$.

- le dépôt de couche monomoléculaire de réactif par la technique de Langmuir-Blodgett.

Les activités biocapteurs incluent les mesures d'électrolytes $\left(\mathrm{K}^{+}, \mathrm{Na}^{+}, \mathrm{Ca}^{2}+\right.$, halogènes, etc...) pour l'analyse médicale, les déterminations enzymatiques par réactions de bioluminescence /47/ (couple NAD-NADH) ou via la mesure de $\mathrm{O}_{2}$ par fluorescence (mesures de glucose, lactate éthanol, cholestérol) $/ 42 /$, les réactions immunologiques spécifiques antigènes-anticorps par variation de fluorescence d'un antigène marqué avec un fluorophore immobilisé en extrémité de fibre.

Les optodes actives intrinsèques utilisent les effets de variation d'indice de réfraction de la gaine optique (effet de gaine pour la détermination de traces d'hydrocarbures dans l'eau) ou les modifications d'interface coeur-gaine (ondes évanescentes, réactions de surface), et les variations de caractéristiques du coeur de la fibre. Dans ce cas on cite souvent l'exemple de la mesure de I'hydrogène avec un montage interférométrique de type Mach Zehnder /48/. Les avantages de l'optique intégrée, des guides d'ondes planaires et des structures génératrices d'ondes évanescentes sont à l'origine de nombreux capteurs biochimiques (immuno-essais) /24/ ou chimiques $/ 49$ / pour lesquels la production de masse est nécessaire.

\section{4. - COMPOSANTS ET SYSTEMES}

Les autres aspects relatifs aux capteurs concernent :

4.1. - Les composants, tels que les sources et détecteurs dont l'état de l'art a été décrit récemment $/ 11,50 \%$, les coupleurs, connecteurs, câbles optiques dont l'état d'avancement n'est pas toujours à la hauteur des besoins industriels et qui parfois constituent un véritable verrou technologique.

4.2. - Le multiplexage, soit en fréquence optique de battement individualisant les capteurs, soit en longueur d'onde (chromatique), soit temporel avec des impulsions par des lignes à retard pour identifier les capteurs ou les lignes de capteurs. En spectrométrie, la mesure multivoies requiert le plus souvent un double multiplexage (spatial) d'une part en amont, d'autre part en aval du point de mesure (figure 14).

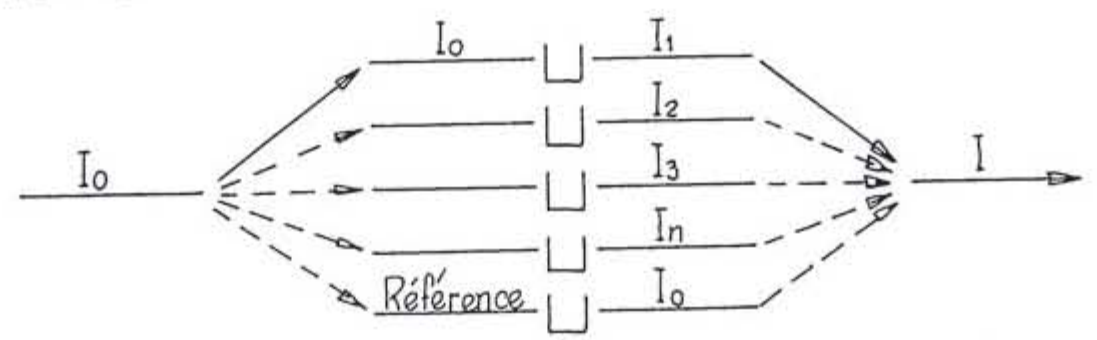

Figure 14 : Exemple de multiplexage spatial pour spectrométrie d'absorption en contrôle de procédé. 
4.3. - Les réseaux de capteurs qui sont une conséquence des possibilités de multiplexage optique : réseau OTDR (figure 15) multiplexé, réseau dispersif en longueur d'onde, réseau interférométrique en lumière blanche, réseau optoalimenté, etc.. A noter que la France a pris un bon départ dans cette voie avec les Société FRAMATOME (Optofra), PHOTONETICS, BERTIN, SPECTEC et CGE.

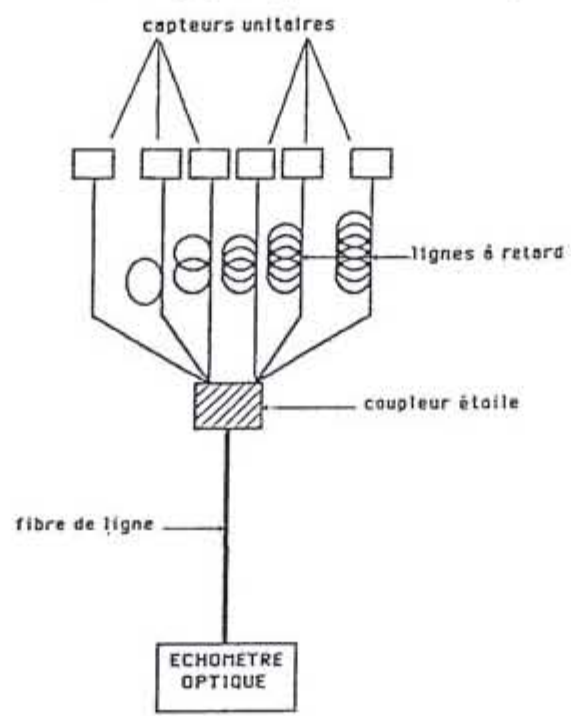

Figure 15 : Réseau de capteurs à multiplexage temporel. Les impulsions de retours des divers capteurs sont décalées temporellement par des lignes à retard ce qui permet de les identifier. Architecture étoile (Photonetics).

\section{CONCLUSION}

Les besoins et applications des capteurs à fibres optiques sont aujourd'hui très ouverts. Ils couvrent les domaines militaires, espace, nucléaire, pétrole, transports, les industries chimiques et parachimiques, les sciences de la vie (médecine, biotechnologies), l'agroalimentaire, le contrôle des procédés industriels, l'environnement, etc... La croissance exponentielle depuis cinq ans des laboratoires de recherches et développements, l'existence en forte augmentation d'industriels qui proposent aujourd'hui des produits, le taux de croissance (évalué à $40 \%$ an par les experts), sont des signes tangibles de l'intérêt de cette nouvelle discipline. II reste cependant encore un long chemin à parcourir pour obtenir des produits "grand public" ou à "faible coutt". Cependant, aujourd'hui, de nombreuses niches clairement identifiées (téléanalyse, etc..) et officiellement reconnues $/ 51$, et l'intérêt croissant des utilisateurs, sont les principaux moteurs de l'action dans ce domaine.

\section{REFERENCES}

/ 1 / GIALLORENZI, T,G ; BUCARO, J,A ; DANDRIDGE, A ; SIGEL, G.J ; COLE, J, H ; RASHLEIGH, S, C; PRIEST, R, G; IEEE J of Quantum Electr. QE 18 (1982) 626.

/ $2 /$ ARDITTY, H, J; BOURBIN, Y ; GRAINDORGE, P; LEFEVRE, H.C. ; Proc. Opto 82, ESI Publ, (1982) 55. 
/ $3 /$ PITT, G, D; EXTANCE, P ; NEAT, R, C ; BATCHELDER, D.N ; JONES, R.E; BARNETT, J,A ; PRATT, R, H: IEEE Proc $132 \mathrm{~J}$ (1985) 214.

/4/ FLUITMAN, J; POPMA, Th; Sensors and Actuators 10 (1986) 25.

/5/ GOURE, J,P; Entropie 155 (1990) 18.

/6/ WOLFBEIS, O, S:

Chapitre 3 dans "Molecular luminescence spectroscopy". Part II, SCHULMAN, S,J (Ed) Wiley and Sons Inc N.Y. (1988) 129.

/7/ SEITZ, W.R., Crit. Rev. Anal. Chem. 19 (1988) 135.

/8/ BOISDE, G; Entropie 155 (1990) 28.

/9/ HARMER, A.L; NARAYANASWAMY, R; Chapitre 13 dans "Chemical Sensors" EDMONS, T,E (Ed), Blackie, G.B. (1988) 275.

/10)/ NORRIS, J, O, W; Analyst 114 (1989) 1359.

/11/ CULSHAW, B; DAKIN, J,P; "Optical Fiber sensors"; Artech House Books Inc. Voll (1988) Vol II (1989).

/12/ WOLFBEIS, O, S ;

"Fiber optic chemical sensors and biosensors" CRC Press Inc. Vol I et II (1991).

/13/ LUBBERS, D, W; OPITZ, N;

Z. Naturforschung $30 \mathrm{C}$ (1975) 532.

/14/ OPITZ, N : LUBBERS, D, W ; Pflugers. Arch. Eur. J. of Physiol 355 (1975) SR 120.

/15/ JACKSON, D, A ; JONES, J, D, C ; Chapitre 10 de la référence 11.

/16/ KERSEY, A, D; DANDRIDGE, A; BURNS, W, K; Electron Lett. 22 (1986)

/17/ EZEKIEL, S; ARDITTY, H,J; "Fiber optic rotation sensors" Springer Verlag Berlin Heidelberg (1981).

/18/ SPILLMAN W, B; KLINE, B, R; MAURICE, L, B; FUCH, P, L; Appl. Opt. 28 (1989) 3166. 
/19/ SANSONETTI, P; LEQUIME, M; GIOVANNINI, H;

OFS 89, Springer Proc. vol. 44 ARDITTY, H, J; DAKIN, J, P ; Kersten R.Th (ed) (1989), 71.

/20/ KOTROTSIOS, G; PARRIAUX, O;

OFS 89 - id, (1989) 75.

/21/ BOISDE, G; BLANC, F; PEREZ, J, J;

TALANTA 35 (1988) 75.

/22/ BERTHOUD, T; FLEUROT, B; HAMON, G; MAUCHIEN, P; Spectra 2000, 120 (1987) 31.

/23/ PLAZA, P; NGUYEN, QUY DAO; JOUAN, M ; FEVRIER, H; SAISSE, H; Analusis 15 (1987) 504.

/24/ PLACE, J, F; SUTHERLAND, R, M ; DAHNE, C; Biosensors 1 (1985) 321.

125/ DESFORGES, $F$, $X$; JEUNHOMME, L, B ; OPTO 88 ESI Public (Ed) (1988) 110.

/26/ GRATTAN, K.T.V. ; Measurement and Control, 22 (1989) 165.

/27/ BOIS, E; HUARD, S, J; BOISDE, G; Appl. Opt. 28 (1989) 419.

/28/ LIU, X, P; SPONCER, R, C; JONES, B, E; Sensors and Actuators A25 (1991) 197.

/29/ "Optoflow", Notice Photonetics (Marly le Roi).

/3O/ KOO, K, P; SIGEL, G, H ; IEEE J., QE18 (1982) 670.

/31/ CHOLLeT, P: Brevet Fr. EN 89.17179 (26.12.89).

/32/ DENAYROLLES, Y; TROUVILLE, B; LEMARQUIS, J, C ; Ann. Telec, 43 (1988) 88.

/33/ FERDINAND, $\mathrm{P}$; Techniques de l'Ingénieur R2800 (1989).

/34/ THEVENIN, J, C; ALlemand, L, R ; CALVEt, J; CAVAN, J.C ; OFS 84 Proc. SPIE Int. Soc. Opt. Eng. 514 (1984) 133.

/35/ BOISDE, G, Résultats non publiés.

/36/ KRONENBERG, S; MC LAUGHLIN, W ; SIEBENTRITT, C, R ; Nucl. Inst. and Methods 190 (1981) 365. 
/37/ BUEKER, H; HAESING, F, W; NICOLAI, ST ; WOLTERS, B; Proc. SPIE Int. Soc. Opt. Eng. 1201 (1990) sous presse.

/38/ PLAZA, P; NGUYEN QUY.DAO ; JOUAN, M ; FEVRIER, H; SAISSE, H ; Appl. Opt. 25 (1986) 3448.

/39/ BOISDE, G; BLANC, F; MAUCHIEN, P ; PEREZ, J.J ; Chapitre 14 dans référence 12.

140/ PETERSON, J, I; GOLDSTEIN, R.V ; FITZGERALD, R, V; BUCKHOLD, D, K ; Anal. Chem. 52 (1980) 864.

/41/ BOISDE, G; BIATRY, B ; MAGNY, B ; DUREAULT, B ; BLANC, F; SEBILLE, B ; Proc. SPIE Int. Soc. Opt. Eng. 1172 (1989) 239.

142/ WOLFBEIS, O.S;

Proc. OFS 89, Springer Verlag Proc. 44 (1989) 416.

143/ BOISDE, G; SEBILLE, B;

Proc. SPIE 1510 (1991) sous presse.

144/ POSCH, H, E; LEINER, M, J.P; WOLFBEIS, O, S; Frezenius Z. Anal. Chem. 334 (1989) 162.

145/ NARAYANASWAMY, R; SEVILLA III, F ; Anal. Chim. Acta 186 (1986) 365.

/46/ JORDAN, D, M ; WALT, D, R; MILANOVICH, F, P ; Anal. Chem. 59 (1987) 437.

/47/ BLUM, L.J; GAUTIER, S, M ; COULET, P, R; Anal. Lett. 21 (1988) 717.

/48/ BUTLER, M, A ; Appl. Phys. Lett. 45 (1984) 1007.

/49/ KREUWEL, H, J, M ; LAMBECK, P, V ; GENT, J.V ; POPMA, Th, J ; Proc. SPIE Int. Soc. Opt. Eng. 798 (1987) 218.

/50/ KIST, R; Dans "Optical Fiber Sensors", CHESTER, A, N ; MARTELLUCCI, S; VERGA SCHEGGI (Eds) Martinus Nijkoff Publishers Série E 132 (1987) 267.

/51/ Rapport du CADAS (Académie Sciences Fr - Paris) Janvier 1991. 